\title{
The role of lumbar puncture in suspected CNS infection-a disappearing skill?
}

\section{R Kneen, T Solomon, R Appleton}

\section{Declining use of lumbar puncture may be detrimental to patient care}

\begin{abstract}
ntroduced by Quincke in 1891 to diagnose and treat tuberculous meningitis, lumbar puncture (LP), has since been used extensively in the investigation of many neurological conditions. ${ }^{1}$ Cerebrospinal fluid (CSF) analysis and culture is the definitive method of diagnosing central nervous system (CNS) infections and, until the late 1970s, all or most patients admitted with suspected CNS infection in Western countries underwent LP. ${ }^{2}$ In many developing countries this remains the practice. However, in the West the use of LP has declined over the last 20 years, primarily because of increasing anxiety that the procedure may precipitate cerebral herniation. In this paper we review the reasons for the declining use of LP and examine whether its usage is now so infrequent that it may be detrimental to patient care.
\end{abstract}

\section{RAISED INTRACRANIAL PRESSURE IN CNS INFECTION}

It has been recognised since the early 1960s that raised intracranial pressure (ICP) is a serious complication of acute bacterial meningitis. ${ }^{3}$ Causes are thought to include occlusion of the arachnoid granulations, cerebritis, generalised brain oedema, inappropriate antidiuretic hormone secretion, subdural effusions or abscesses, seizures, and an increase in CSF production. ${ }^{4}$ Raised ICP in itself does not cause brainstem herniationpatients with idiopathic (benign) intracranial hypertension may have very high pressures without causing herniation or death. In other CNS infections, including cerebral malaria, high opening pressures are often found, but herniation is rare. ${ }^{5}$

Herniation can result when raised ICP leads to differences in pressure between different brain compartments. ${ }^{6}$ The uncus of the temporal lobe may herniate through the tentorial opening, or the cerebellar tonsils may herniate through the foramen magnum. These processes damage the brainstem either directly or indirectly, by compromising its blood supply resulting in ischaemia and haemorrhage. By careful examination, it is possible to recognise the different herniation syndromes and rostro-caudal progression from one to the next (see fig 1$).^{78}$

\section{DOES LUMBAR PUNCTURE CAUSE CEREBRAL HERNIATION?}

Initial concern about the safety of LP in children with bacterial meningitis was raised in 1978 following the publication of a retrospective review of more than 300 children with proven bacterial meningitis, 10 of whom died. ${ }^{9}$ Eighteen children had an episode of cerebral herniation diagnosed clinically. Two of the 10 deaths were attributed to this mechanism and in one patient cerebral oedema and uncal herniation were confirmed at autopsy. One patient had clinical signs of herniation immediately following LP, which (consequently) led the authors to consider whether LPs contributed to herniation.

In 1982, a report from the Royal College of Physicians (UK) reviewed 88 of the 90 deaths from meningococcal infection (including septicaemia) in England and Wales during $1978 .{ }^{10}$ Coning was diagnosed clinically in six meningitis cases and confirmed at autopsy in five. Five of the six patients had undergone LP, but one patient (with autopsy evidence of coning) died before LP was performed. The authors concluded that LP could precipitate coning and suggested that LP may not be mandatory in all cases of clinically obvious meningococcal infection.

\section{CONSENSUS}

The debate on the safety of LP continued following the report of a 20 month old child with meningococcal meningitis who had a respiratory arrest two hours after LP, and died 48 hours later. ${ }^{11}$ Coning was not confirmed because there was no autopsy. Four experts were asked to comment on whether LPs should be delayed in all "sick" children; two considered that LP should be avoided if there was a clear clinical diagnosis and two felt that LP should still be undertaken. ${ }^{11}$ Subsequent correspondence highlighted the lack of available guidelines for undertaking LP and specifically citing indications and contraindications. ${ }^{12} \mathrm{~A}$ personal view on the contraindications to undertaking LP suggested that LP should be avoided in sick patients with signs of raised ICP who might be at imminent risk of cerebral herniation. ${ }^{13}$ These were similar to recommendations produced by a comprehensive review on meningitis from the USA. ${ }^{14}$ Although there was no evidence that LP caused herniation in patients who would not have herniated anyway, a working consensus was achieved, which appeared to change little over the past 15 years. ${ }^{73-17}$ In summary, it was generally recommended that LP should be undertaken on all patients with suspected CNS infection unless one of the following contraindications is present:

- signs of raised intracranial pressurealtered pupillary responses, absent Doll's eye reflex, decerebrate or decorticate posturing, abnormal respiratory pattern, papilloedema, hypertension and bradycardia

- recent (within 30 minutes) or prolonged (over 30 minutes) convulsive seizures

- focal or tonic seizures

- other focal neurological signs-hemi/ monoparesis, extensor plantar responses, ocular palsies

- Glasgow Coma Score $<13$ or deteriorating level of consciousness

- strong suspicion of meningococcal infection (typical purpuric rash in an ill child)

- state of shock

- local superficial infection

- coagulation disorder

Although not previously defined, the definition of a prolonged seizure used here (more than 30 minutes) is the currently accepted definition of convulsive status epilepticus. ${ }^{18}$ Some debate exists as to whether a GCS of less than 13 or less than 12 is the most appropriate cut off as a contraindication to undertaking LP. $^{715}$ Decerebrate posturing, misdiagnosed as tonic seizures, may be a manifestation of raised intracranial pressure and focal seizures may potentially indicate a structural abnormality including a tumour or an abscess. The occurrence of tonic-clonic, myoclonic, absence, and atonic seizures, in isolation, do not represent contraindications for LP.

\section{CONFUSION}

In 1992 the issue became confused with the publication of a retrospective case review of 445 children with bacterial meningitis admitted to an Australian teaching hospital between 1984 and 1989, which examined the relationship between LP and herniation. ${ }^{19}$ Nineteen 


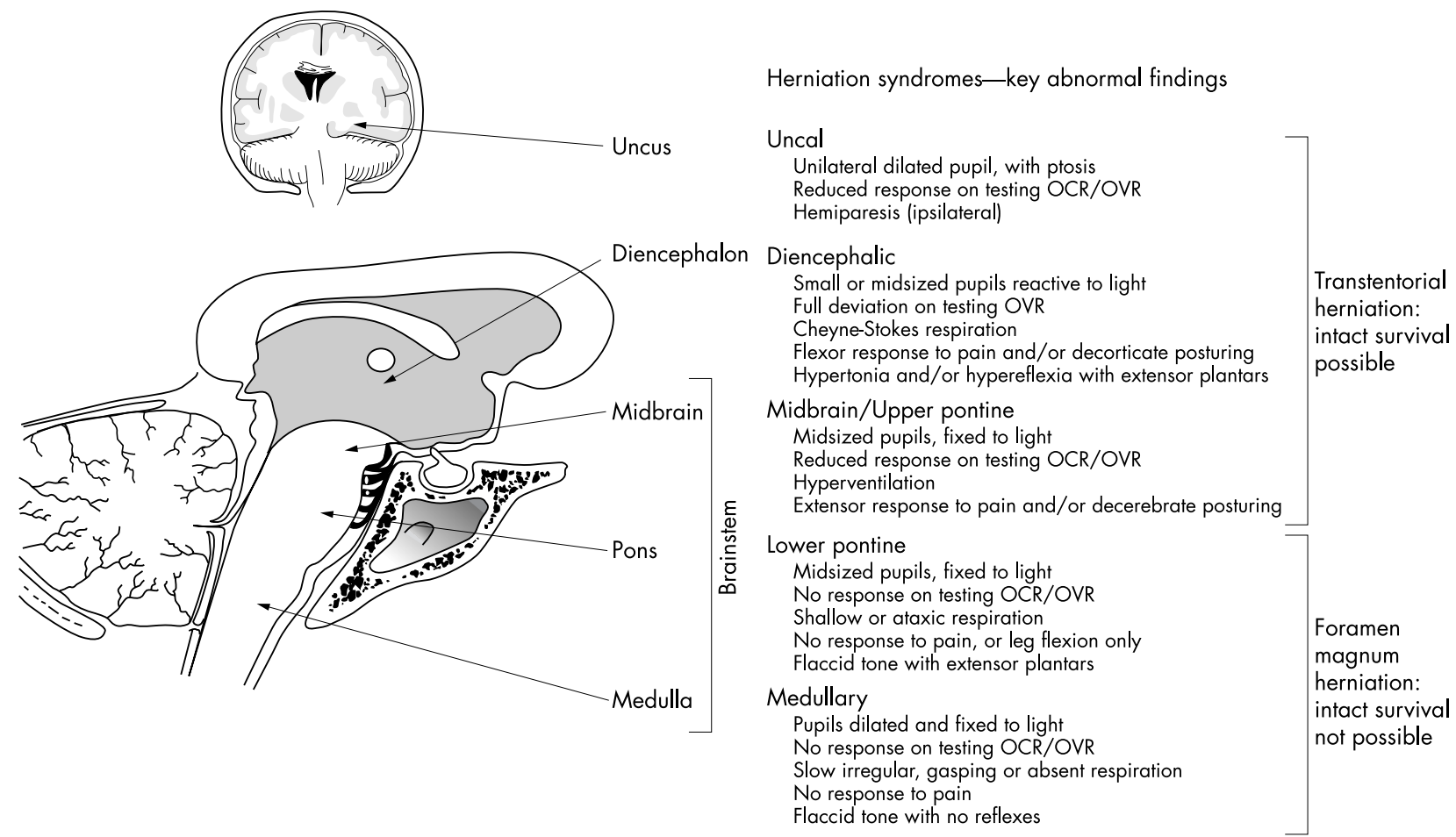

Clinical signs in brainstem herniation syndromes. Coronal (upper) and mid-sagittal (lower) sections of the brain highlighting the neuroanatomy and principal clinical manifestations of herniation syndromes. OCR, oculocephalic (doll's eye) reflex; OVR, oculovestibular (caloric) reflex. (Reproduced with permission from reference 6.)

children had 21 clinically diagnosed episodes of coning of whom 14 died, two before undergoing LP. Of the remaining 19 coning episodes, three occurred before LP was undertaken, eight had an "early coning episode" (within three hours of LP) and eight occurred between three and 37 hours after LP. Autopsies in six patients revealed clear evidence of cerebral herniation in five and equivocal evidence in one patient. In view of the apparent cluster of early coning herniation episodes, the authors concluded that a clear temporal relationship existed between LP and cerebral herniation, and that LP could therefore cause herniation. However, as subsequent correspondence emphasised, a temporal relationship does not necessarily imply causality. ${ }^{20}{ }^{21}$ An analysis of the relationship between blood cultures and herniation from the same study would also have shown a similar, and apparently positive relationship. More importantly, the study showed that many of these patients would not have undergone LP had the recommended contraindications been in practice. However, instead of being used to reinforce the recommendations, most clinicians appeared to interpret the findings of this study as further evidence against the use of LPs in any patient with suspected CNS infection.

The same year saw the publication of a review of 252 patients with meningococcal disease, including septicaemia, diagnosed in Gloucestershire over a 14 year period. ${ }^{22}$
Two of 17 deaths were attributed to a "neurological deterioration" after LP, with herniation confirmed at autopsy, although it was not stated whether there was clinical evidence of herniation before LP. Two other deaths were attributed to a delay in antibiotic treatment following normal initial CSF findings. The authors critically questioned the need for LP in patients with meningococcal meningitis but correspondents critically questioned the clinicans caring for the patients. It is well recognised that normal early CSF findings do not necessarily exclude bacterial meningitis and for this reason antibiotics should be given until CSF cultures are negative in those children who are felt to have a clinical diagnosis of meningitis. ${ }^{23}$

\section{THE LIVERPOOL EXPERIENCE}

Despite the reservations about the Gloucester paper's conclusions, ${ }^{23}$ and although it only considered patients with meningococcal disease (ignoring all other CNS bacterial and viral infections), its message became inappropriately applied to all patients with suspected CNS infection. Consequently, it would appear that many doctors have stopped undertaking LPs in patients with suspected CNS infection. In our own hospital a four month case note review of 448 acute medical admissions revealed that LP was performed in only $53 \%$ of 47 children who had clinical evidence or suspicion of CNS infection, and with no medical contraindications (Kneen R, Solomon T,
Appleton R, unpublished observations). None of the patients who underwent LP experienced a neurological deterioration including recognisable cerebral herniation. In addition, only 17 of the 25 patients who had LP, CSF analysis was incomplete or inadequate-for example, no measurement of CSF glucose or protein levels, no simultaneous measurement of blood glucose levels, or no CSF cultures. Discussion with colleagues throughout the country has shown that our experience is not unique (Kneen $\mathrm{R}$ and Appleton R, personal communications). At some centres, no LP is performed without first consulting a paediatric neurologist, and at others, junior doctors believe LPs should never be performed without a CT scan-even in fully conscious patients with no neurological signs-despite the well recognised fact that a normal CT scan does not necessarily exclude raised ICP. Clinical signs remain the best predictors of incipient uncal herniation. ${ }^{24}$

In addition to concerns about safety, many doctors believe that because of newer diagnostic techniques (including specifically the polymerase chain reaction (PCR)) for bacterial antigens and because all children with possible meningitis or encephalitis receive antibiotics and/or aciclovir routinely, LP would contribute little to their management. However, our case note review of acute paediatric admissions showed that results of 
LPs contributed directly to patient management in $72 \%$ of cases, either by identifying an organism, allowing unnecessary antibiotics to be stopped after 24 hours, or by permitting an earlier discharge from hospital.

The reduction in the number of LPs being performed by junior doctors, with or without advice from their senior colleagues, may have a number of consequences. What was previously considered to be a routine, potentially important, and relatively safe investigation is now becoming almost obsolete. Whereas 10 years ago most doctors learnt to perform LPs as medical students or house officers, there are now many senior house officers who have never performed an LP, or even seen one being undertaken. The recent trend towards ward based and shift work, where juniors often do not follow up patients they admit, has meant they are less likely to see the benefits of investigating patients fully. It is easier to prescribe antibiotics and then "forget about the patient", rather than perform LP, chase up the result, and then make an informed decision about the management. It is only when this strategy fails and patients have an unusual illness course or persisting non-specific symptoms and/or pyrexia that the benefit of an early LP is appreciated.

The advantages of obtaining a microbiological diagnosis extend beyond individual patient management. Identifying an organism allows appropriate prophylaxis to be recommended for close contacts, and public health service monitoring for disease outbreaks. Finally, there are potential health economic implications with reduced antibiotic and/or aciclovir usage and shorter hospital admissions for those children in whom CSF analysis has excluded meningitis or meningoencephalitis.

In summary, the circumstances in which LP should not be performedpossible incipient herniation, episodes of abnormal posturing including tonic seizures, and obvious meningococcal disease-have been clearly defined. There is no evidence that the procedure is harmful in most other patients with suspected CNS infection. We believe that in the majority of these patients LP is safe, and potentially beneficial. In contrast, the avoidance of LP would appear to be based on unsound and anecdotal evidence and may be potentially detrimental to patient care.

Arch Dis Child 2002;87:181-183

\section{Authors' affiliations}

R Kneen, T Solomon, R Appleton, The Roald Dahl EEG Unit, Department of Neurology, Royal Liverpool Children's Hospital (Alder Hey), Liverpool L12 2AP, UK

Correspondence to: Dr Appleton

Richard.Appleton@RLCH-TR.NWEST.NHS.UK

\section{REFERENCES}

1 Gorelick PB, Biller J. Lumbar puncture: technique, indications, and complications. Postgrad Med 1986:79:257-68.

2 American College of Physicians. Health and Public Policy Committee. The diagnostic spinal tap. Ann Intern Med 1986;104:880-5.

3 Dodge $\mathbf{P}$, Swartz M. Bacterial meningitis. A review of selected aspects. N Eng J Med 1965:272:898-902.

4 Minns RA, Engleman HM, Stirling $\mathrm{H}$. Cerebospinal fluid pressure in pyogenic meningitis. Arch Dis Child 1989;64:814-20.

5 Waller D, Krishna S, Crawley J, et al. Clinical features and outcome of severe malaria in Gambian children. Clin Infect Dis 1995;21:577-87.
6 Plum F, Posner JB. The pathological physiology of signs and symptoms in coma In: Plum F, Posner JB, eds. The diagnosis of stupor and coma. Philadelphia: FA Davis Co, 1982:1-73.

7 Kirkham FJ. Non-traumatic coma in children. Arch Dis Child 2001;85:303-12.

8 Solomon T. Neurological Presentations. In: Beeching N, Gill G, eds. Lecture Notes on Tropical Medicine. Oxford: Blackwell Science 2002

9 Horwitz SJ, Boxerbaum B, O'Bell J. Cerebral herniation in bacterial meningitis in childhood. Ann Neurol 1978;7:524-8.

10 Slack J. Deaths from Meningococcal infection in England and Wales in 1978. J R Coll Physicians Lond 1982;16:40-4.

11 Harper JR. Timing of lumbar puncture in severe childhood meningitis. BM 1985;291:651-2.

12 Spender $\mathbf{Q}$. Timing of lumbar puncture in childhood meningitis. BN 1985;291:898.

13 Addy D. When not to do a lumbar puncture. Arch Dis Child 1987;62:873-5

14 Klein JO, Feigin RD, McCracken Jr GH. Report of the Task Force on Diagnosis and Management of Meningitis. Pediatrics 1986;78:959-82.

15 Advanced Life Support Group. Advanced Paediatric Life Support, 2nd ed. London: BM Paediatric Life Support, 2
Publishing Group, 1997.

16 Mellor D. The place of computed tomography and lumbar puncture in suspected meningitis. Arch Dis Child 1992;67:1417-9.

17 Pollard AJ, Britto J, Nadel S, et al. Emergency management of meningococcal disease. Arch Dis Child 1999;80:290-6.

18 Appleton R, Choonara I, Martland T, et al. The treatment of convulsive status epilepticus in children. The Status Epilepticus Working Group. Arch Dis Child 2000;83:415-9.

19 Rennick G, Shann F, de Campo J. Cerebral herniation during bacterial meningitis in children. BM 1993;306:953-5

20 Obaro SK. Avoiding coning in childhood meningitis. BN 1993;306:1692.

21 Jones SW, Webb D. Cerebral herniation in bacterial meningitis. BM 1993;306:1413.

22 Wylie PAL, Stevens D, Drake III W, et al. Epidemiological and clinical management of meningococcal disease in West Gloustershire: retrospective, population based study. BM 1997;315:774-9.

23 Stephenson T. Coning may occur without lumbar puncture being done. BM 1998;16:1015.

24 Cabral DA, Flodmark O, Farrell K, et al. Prospective study of computed tomography in acute meningitis. J Pediatr 1987;111:201-5. 


\section{LETTER}

If you have a burning desire to respond to a paper published in $A D C$ or $F \mathcal{N}$, why not make use of our "rapid response" option?

Log on to our website (www.archdischild. com), find the paper that interests you, click on "full text" and send your response by email by clicking on "submit a response".

Providing it isn't libellous or obscene, it will be posted within seven days. You can retrieve it by clicking on "read eLetters" on our homepage.

The editors will decide, as before, whether to also publish it in a future paper issue.

\section{Mortality in meningococcal disease: please report the figures accurately}

We read with great interest the two recent articles on mortality in meningococcal disease. $^{12}$ While we would agree with the message contained in both articles, namely that the mortality associated with this condition has decreased with time, we have serious concerns regarding the presentation of the data in the paper from the St Mary's group.

Booy and colleagues report a crude mortality of $2 \%$ for the year $1997,{ }^{2}$ a figure that has generated considerable media interest. Several reasons are cited for this falling mortality: the provision of mobile intensive care, meticulous attention to stabilising the patient whilst in the district hospital, and the existence of a specialist "sepsis" intensive care unit. However the way in which the mortality data are presented demonstrate several contradictions. Booy and colleagues purport that intensive care begins from the time the retrieval team is contacted, indeed they calculate PRISM mortality risk from this time, yet mortality is calculated only from those patients who physically arrive back in their own unit. It is well known that mortality from meningococcal disease is greatest in the first 6 hours, primarily from myocardial failure. ${ }^{3}$ To cull non-survivors before PICU admission thus creates a self fulfilling prophesy.

Table 1 Mortality data for severe meningococcal patients retrieved to Guy's Hospital January 1998 to November 2001

\begin{tabular}{|c|c|c|c|c|}
\hline & 1998 & 1999 & 2000 & 2001 \\
\hline $\begin{array}{l}\text { Deaths prior to team arrival } \\
\text { Time to death from PICU team arrival }\end{array}$ & 1 & 1 & 1 & 1 \\
\hline Less than 6 hours & 3 & 2 & 1 & 1 \\
\hline 6 to 12 hours & 0 & 0 & 1 & 1 \\
\hline 12 to 24 hours & 2 & 0 & 0 & 0 \\
\hline Greater than 24 hours & 0 & 0 & 0 & 0 \\
\hline Total PICU deaths & 5 & 2 & 2 & 2 \\
\hline Total survivors & 40 & 45 & 56 & 31 \\
\hline
\end{tabular}

To illustrate this, we present data from our own unit (Guy's Hospital), which cover a similar geographical catchment area to St Mary's. The table shows mortality data for the period January 1998 to November 2001, illustrating time of death after arrival of the retrieval team. For the sake of completeness, we have also included the four deaths that occurred before the arrival of the team. Over this period we have undertaken 183 retrievals on patients with severe meningococcal disease; $147(80 \%)$ of these required mechanical ventilation and/or inotropic support. $12 \%$ of these patients presented with meningitis alone, the remainder with septic shock. The overall crude mortality is $8.2 \%$ (15/183), which includes four patients who died before arrival of the retrieval team. Our death rate becomes comparable to that of St Mary's if we exclude patients who die within 6 hours of the retrieval team's arrival, producing a mortality of $4 / 176(2.3 \%)$

Our unit policy is one of rapid stabilisation before transfer, as evidenced by a median time spent out of the PICU (the sum of the time spent in the district general hospital and the transit time back to PICU) of 2 hours $35 \mathrm{~min}$ utes. This resulted in only one death in the district general hospital, none during transfer, but a considerable proportion in the early hours following PICU admission.

It is our impression that the St Mary's retrieval process is a considerably longer one, which may artifactually reduce PICU mortality. We would therefore ask that the St Mary's group present their data in a similar fashion, including retrieval times. Specifically, were the 29 deaths before physical admission to the PICU occurring whilst under the management of the retrieval team at the local hospital (and thus under PICU management, by their own definition)? If so, mortality should be adjusted accordingly. Second, has this trend continued in subsequent years? This disease attracts media and public attention par excellence. It is therefore vital that outcome data which are accessible to the public and may be used to influence service reorganisation be presented in a transparent manner

S M Tibby, I A Murdoch, A Durward

Department of Paediatric Intensive Care, Guy's Hospital, St Thomas Street, London SE I 9RT, UK

Correspondence to Dr Tibbey ShaneTibby@gstt.sthames.nhs.uk

\section{References}

1 Thorburn K, Baines $\mathrm{P}$, Thomson, Hart CA. Mortality in severe meningococcal disease. Arch Dis Child 2001;85:382-5.

2 Booy R, Habibi P, Nadel S, et al. Reduction in case fatality rate from meningococcal disease associated with improved healthcare delivery. Arch Dis Child 2001;85:386-90.

3 Mercier JC, Beaufils F, Hartmann JF, et al. Hemodynamic patterns of meningococcal shock in children. Crit Care Med

$1988: 16: 27-33$

\section{Inter-unit comparisons are flawed}

Mortality from meningococcal septic shock may be falling; however, it is difficult to be sure. Inter-unit comparisons of the sort precipitated by these articles and correspondence are inevitably distorted by confounding factors. These factors are not entirely removed by the use of mortality prediction models.

Historically, mortality data for meningococcal septicaemia from the UK Public Health Laboratory Service Communicable Disease Surveillance Centre have always shown a lower mortality rate than that in many paediatric intensive care units. However, the comparison is regarded as inappropriate because the surveillance data include patients with positive blood cultures (septicaemia) who were not shocked and so would be expected to survive without intensive care. If one admits such patients to intensive care then both crude and standardised mortality are artificially reduced. Furthermore, mortality rates from individual intensive care units or time periods are difficult to compare even using mortality prediction models, without reassurance that the same threshold for admission and/or intervention applies in each case.

The paper by Booy et al contains no reassurances on this issue and no information is given about the performance of the mortality prediction model (PRISM) on their data. Furthermore their series includes mortality rates that appear to exclude the deaths during retrieval. This despite the fact that the quality of retrieval is hailed as a potential cause of decreased mortality. Thorburn et al provide some reassurance by quoting a consistently high rate of ventilation in the reported cases and detailed information on the performance of the mortality prediction model. Hence if there has been a decrease in the threshold for admission it has been accompanied by an increased use of ventilation and perhaps other interventions. It is not clear whether the data from the north west include deaths during retrieval, prior to admission to the PICU.

Both series significantly outperform the expected mortality predicted by PRISM which is not surprising and calls into question the use of the model. Convincing evidence of a fall in mortality for meningococcal septic shock however requires a uniform definition of the illness and "all cause" mortality data from a geographically defined resident population. The regional arrangement for delivery of paediatric intensive care in the north west of England combined with the factors mentioned above make it far more likely that Thorburn et al have indeed detected a true improvement in survival for this condition. Since 1996 there has been a trend for more 
children to receive intensive care in lead centres $^{12}$ and this might be expected to reduce mortality across the board.

G Pearson Paediatric Intensive Care Unit, Birmingham Children's Hospital, Steelhouse Lane, Birmingham B4 6NH, UK

Gale.Pearson@Bhamchildrens.wmids.nhs.uk

\section{References}

1 Pearson GA, Shann F, Barry P, et al. Should intensive care be centralised? Trent versus Victoria Lancet 1997:349:1213-17.

2 Pearson G, Barry P, Timmins C, et al. Changes in the profile of paediatric intensive care associated with centralisation. Intensive Care Med 2001;27:1670-3.

\section{Improved outcome in severe meningococcal disease}

We thank Tibby et al and Pearson for their interest in our paper. ${ }^{1}$ We agree with Pearson that evidence for a fall in overall mortality in meningococcal septic shock would require a geographical community based study. We described mortality in severe meningococcal disease in a paediatric intensive care unit (PICU)

In our multispeciality PICU in the north west, we have observed a continued decrease in both actual PICU mortality and mortality adjusted for disease severity since the original study period (table 1). Paediatric index of mortality (PIM) is a more contemporary scoring system than PRISM (paediatric risk of mortality score), and so has been calibrated to the more recent decline in PICU mortality rates. PIM gives a score at point of first PICU contact.

This general trend of improving meningococcal outcome is also reflected in other PICUs. As shown by the results from St Mary's PICU in London, where in a group with an overall actual mortality of $18.7 \%$ (PICU mortality for the study period being $10 \%$, and an additional $8.7 \%$ mortality for the "unretrievables"), they encouragingly had managed to reduce the meningococcal PICU mortality in their "specialist PICU" from 23\% to 2\% (1992-97). ${ }^{3}$ Tibby et al, from Guy's Hospital PICU in London (1998-2001), in their letter report a similar very low mortality rate.

There has been continued improvement in outcome from severe meningococcal disease throughout the UK. Early recognition and early institution of treatment are of paramount importance. No single centre holds the monopoly on the improved outcome in meningococcal disease. Although improved intensive care has undoubtedly contributed to this fall in mortality, there should be more recognition of the role of those in the community, parents and carers, general practitioners, and district general hospitals who have significantly contributed (and continue to contribute) to the survival of these critically ill children.

K Thorburn, A Thomson, A Hart Royal Liverpool Children's Hospital Alder Hey, UK

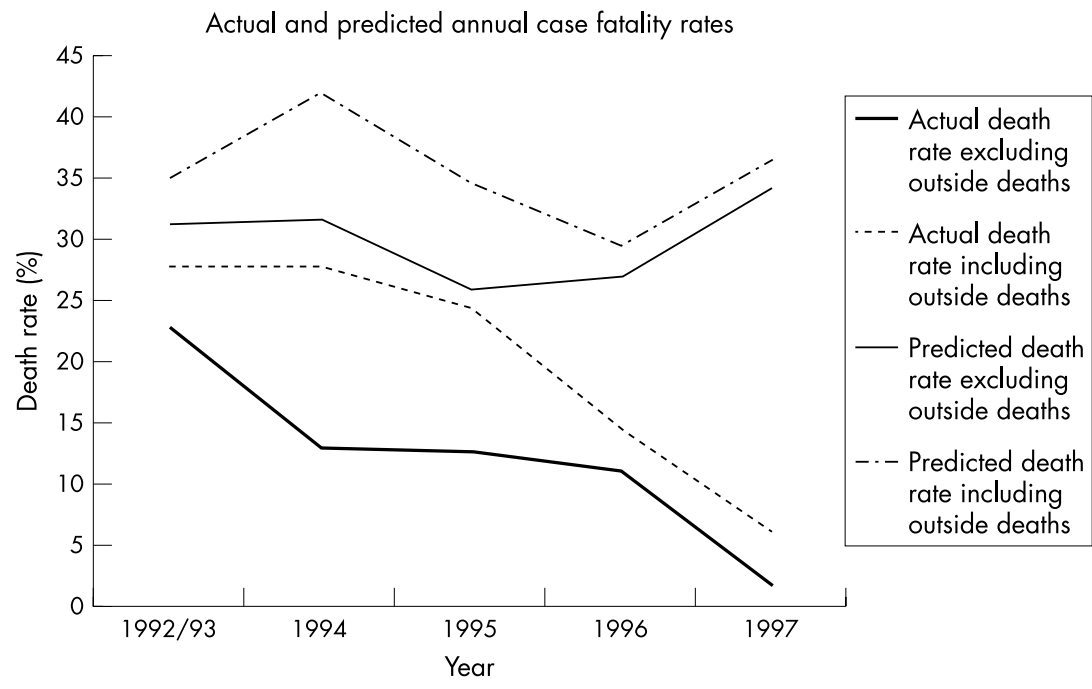

Figure Actual and predicted annual case fatality rates.

\section{References}

1 Thorburn K, Baines P, Thomson A, et al. Mortality in severe meningococcal disease. Arch Dis Child 2001;85:382-5.

2 Shann F, Pearson G, Slater A, et al. Paediatric index of mortality (PIM): a mortality prediction model for children in intensive care. Intensive Care Med 1997;23:201-7.

3 Booy R, Habibi P, Nadel S, et al. Reduction in case fatality from meningococcal disease associated with improved healthcare delivery. Arch Dis Child 2001;85:386-90

\section{Mortality in meningococcal disease: please report the figures accurately}

We thank Tibby and colleagues for their interest. We believe they and others would be interested in the accompanying figure (see above)

It compares yearly case fatality rates on all referrals to St Mary's PICU, regardless of whether they died before a mobile intensive care team arrived or while the team was assisting with resuscitation. The 29 "outside" deaths are included ( 3 in 1992/3, 8 in 1994, 10 in 1995, 3 in 1996, 5 in 1997). As stated in our published paper, logistic regression analysis, controlling for disease severity, age and sex, and including these extra deaths, showed no change in the estimated odds ratio for the yearly reduction in death rate, namely 0.41 . The overall case fatality rate for 1997 became $6 \%$ compared with the PICU admission rate of $2 \%$ and a predicted case fatality rate $34 \%$ using PRISM scores.

For the 5 deaths in 1997 outside St Mary's PICU, response times between call to the unit and arrival of a team at the DGH varied between 100 and 185 minutes. One child died
Table 1 Actual mortality, number of patients, mortality per year, and standardised mortality ratio (SMR) in patients admitted to the paediatric intensive care unit (PICU) at the Royal Liverpool Children's Hospital with meningococcal disease

Jan 1995 to March $1998^{1} \quad$ April 1999 to March 2001

$\begin{array}{lll}\text { Actual mortality/PICU admissions } & 11 / 123(8.9 \%) & 3 / 95(3 \%) \\ \text { Mortality per year } & 3.5 & 1.5 \\ \text { PIM predicted SMR } & 1.16 & 0.24\end{array}$

PIM predicted SMR

1.16

0.24

SMR = paediatric index of mortality (PIM) predicted mortality/actual mortality. as the local hospital were telephoning us, two arrested within 90 minutes of St Mary's being called and died within minutes of the team arriving, and the other two died between 2 and 7 hours after arrival.

R Booy

Department of Paediatrics, Imperial College School of Medicine, St Mary's Hospital, Norfolk Place, London W2 IPG, UK; r.booy@qmul.ac.uk

\section{Genuine reduction in meningococcal deaths results from teamwork}

As paediatric intensivists in lead centres accredited for paediatric intensive care (PIC) training and responsible for the care of approximately 7000 cases per year, we read with concern the report from St Mary's Hospital which reported improved outcome of meningococcal disease (MD) in 1997 compared with previous years.

Their reported reduction in mortality must be seen in the context of an overall reduction of childhood mortality and a widespread improvement in the outcome for many conditions requiring PIC such as acute respiratory failure, $^{2}$ persistent pulmonary hypertension ${ }^{3}$ and complex congenital heart defects. ${ }^{4}$ Overall UK PIC mortality rates have fallen to a standardised mortality ratio (SMR) of 0.87 as compared with the model generated in $1994 .^{6}$

Their application of the severity of illness score (PRISM) is incorrect. No patient has a $100 \%$ predicted risk of mortality and therefore all deaths observed in any such study must increase the SMR. The exclusion of nearly half of the total deaths $(29 / 62,47 \%)$ who did not survive the long stabilisation and overall retrieval times must reduce SMR regardless of any other intervention. Whilst inclusion of these cases does not alter the direction of the relationship between SMR and year, it raises the overall mortality in the series towards $20 \%$ and more than doubles the headline mortality in 1997. Data from the last 4 years would be of interest. In addition, the lack of any data relating to the performance of the model in different risk groups fails to address the potential confounding factor of disease severity. Since all survivors will reduce SMR, one cause of apparent improvement in risk-adjusted survival is increased admission of low risk cases. assessed by the Paediatric Index of Mortality ${ }^{5}$ 
Recent series from other institutions have followed the convention of presenting data by level of predicted risk. ${ }^{7-9}$

The claim that their "Mobile Intensive Care" service is the key element in improved survival is confusing when all the cases that died under the care of this service were excluded from both the analysis and the "headline" figure of $2 \%$ mortality for MD.

However, our greatest concern is the claim that these data support their particular "model" of care of critically ill children. This is not consistent with their report, as St Mary's had been performing transports since 1992 but the fall in mortality occurred some $4-5$ years later. It should be remembered that PICU retrievals have been performed in Liverpool and Glasgow since the late 1970s. Their claim that this "model" has reduced mortality of meningococcal disease is also inconsistent with the similar improvements in outcome presented by other PICs. ${ }^{7-9}$

We feel the narrow focus of the paper on the ICU care of MD is misleading. It ignores the important contribution of many others including parents, charities, and healthcare workers Their role in education, early identification, treatment, and immediate high quality resuscitation is discounted. To imply that ICU management after the initial resuscitation is the key factor in improved survival undermines the vital contributions of these groups.

M Peters, A Petros

Paediatric Intensive Care Unit, Great Ormond St Hospital, Great Ormond Street, London WCIN 3JH, UK

P Baines

Paediatric Intensive Care Unit, Royal Liverpoo Childrens' Hospital, UK

$P$ Loan

Paediatric Intensive Care Unit, Royal Belfast Hospital for Sick Children, UK

P Cullen

Paediatric Intensive Care Unit, Royal Hospital for Children, Glasgow, UK

C Ralston

Paediatric Intensive Care Unit, Birmingham Children's Hospital, UK

R Yates

Paediatric Intensive Care Unit, Manchester Children's Hospital, UK

M Marsh

Paediatric Intensive Care Unit, Southampton General Hospital, UK

P Weir

Paediatric Intensive Care Unit, St Michael's Hospital, Bristol, UK

Correspondence to: A J Petros, Great Ormond Street Hospital, Great Ormond Street, London, UK petroa@gosh.nhs.uk

\section{References}

1 Booy R, Habibi P, Nadel S, et al. Reduction in case fatality rate from meningococcal disease associated with improved healthcare delivery. Arch Dis Child 2001;85:386-90.

2 Peters MJ, Tasker RC, Kiff KM, et al. Acute hypoxemic respiratory failure in children: case mix and the utility of respiratory severity indices. Intensive Care Med 1998;24:699-705.

3 UK collaborative randomised trial of neonatal extracorporeal membrane oxygenation. UK Collaborative ECMO Trail

Group. Lancet 1996;348:75-82.

4 Andrews R, Tulloh R, Sharland G, et al. Outcome of staged reconstructive surgery for hypoplastic left heart syndrome following antenatal diagnosis Arch Dis Child 2001;85:474-7.
5 Pearson GA, Stickley J, Shann F. Calibration of the paediatric index of mortality in UK paediatric intensive care units. Arch Dis Child 2001;84:125-8.

6 Shann F, Pearson G, Slater A, et al. Paediatric index of mortality (PIM): a mortality prediction model for children in intensive care. Intensive Care Med 1997;23:201-7.

7 Thorburn K, Baines P, Thomson A, et al. Mortality in severe meningococcal disease. Arch Dis Child 2001:85:382-5.

8 Peters MJ, Ross-Russell RI, White D, et al. Early severe neutropenia and thrombocytopenia identifies the highest risk cases of acute meningococcal disease. Ped Crit Care Med 2001;2:225-31.

9 Tibby, S. M. Mortality in meningococcal disease: please report the figures accurately http://adc.bmijournals.com/cgi/eletters/ archdischild;85/5/386\#224. 26-10-2001.

\section{Reduction in case fatality rate from meningococcal disease is due to genuine teamwork}

We read with disappointment the response of Dr Peters and colleagues ${ }^{1}$ to our article "Reduction in case fatality rate from meningococcal disease associated with improved healthcare delivery". ${ }^{2}$ It is unfortunate that there appears to be a misunderstanding of the message of our study which demonstrated a significant improvement in the mortality of children with meningococcal disease (MD) over a period of time. Contrary to their concerns those results were achieved through genuine teamwork" as stated in our paper.

In answer to the specific points they raised: We and other intensivists are also aware that mortality in conditions other than MD is also improving. In our paper we did not state that MD was the only condition in which there is an improvement in mortality. Our paper referred to a study published in Critical Care Medicine which also showed improving survival rates of paediatric patients (with various diseases) over time in another paediatric intensive care (PIC) setting. ${ }^{3}$

With reference to the patients who died at the referring hospital and their exclusion from the study. Our paper clearly states "Logistic regression analysis, controlling for disease severity, age and sex, showed that over the study period (1992-97) the overall estimate for the reduction in the odds of death was $59 \%$ per year (odds ratio for the yearly trend $0.41,95 \%$ CI $p=0.000001)$. This estimate and significance remained the same after inclusion of the 29 deaths that occurred at local hospitals".

We did not claim that mobile intensive care is the key element in improved survival. What we stated was: "Considerable changes in the management of patients with MD have occurred over the study period. While no single factor alone is likely to explain the reduction in mortality, several factors might have contributed to the improved outcome. In the past, few centres, including those with PICUs, admitted more than a small number of patients with MD annually. Furthermore, patients were often considered too sick to transfer to a specialist centre and were treated in the A\&E department, paediatric ward or adult ICU of the local district general hospital. Establishment of a mobile intensive care team allowed the centralisation of care of children with MD at a specialist clinical and research unit, which in turn enabled extensive experience in the management of MD to be developed; this may be the most important reason for the improved outcome.... In other words, it was the increased experience in dealing with meningococcal disease that was the critical factor.

The role of mobile intensive care was more directly addressed when we stated that it "has probably been another important factor in improved outcome", not the key factor.

The conclusions of our paper clearly state the multiple factors responsible for the results of the study, which have shown that a notable reduction in the case fatality rate for $\mathrm{MD}$ has been achieved.

The purpose of presenting our data was to emphasise the improvements in mortality in a particular group of patients brought about by a change in health care delivery. The key point being early intervention by a multidisciplinary team with a major research interest in the care of the critically ill child with infectious disease, who have the benefit of a "critical mass" experience.

The PICU at St Mary's Hospital, London was established in 1992, at the time primarily to facilitate the enrolment of children with meningococcal disease into clinical trials. As a large number of critically ill children were referred to our unit, we were subsequently able to record high-quality data regarding clinical status, severity of illness and outcome. We began to demonstrate a reduction in mortality from 1994 onwards, as it takes time to establish the clinical experience which can have a significant impact on the disease process.

The unit at St Mary's has been greatly involved in the development of a model of care involving "genuine teamwork" with the aim of improving the healthcare of children with MD. To this end we have been working with the meningitis charities which are acknowledged on the paper) and other agencies to develop guidelines, publish treatment algorithms and improve policies. In addition our research unit has played a key role in the design and implementation of clinical trials of adjunctive treatments in meningococcal disease, which has led to the publication of the only two large randomised, double blind, placebo controlled studies in childhood septic shock. $^{45}$

Finally we are humbled by the magnitude of response from many other colleagues who have applauded our efforts. We believe, and have repeatedly stated, that what has been widely accepted as a major advance in the outcome of children with MD, could only have been achieved by multidisciplinary effort involving all sectors of health care delivery.

S Nadel
P Habibi
C de-Munter
J Britto
M Levin
Department of Paediatrics, St Mary's Hospital,
London W2 1NY, UK

Correspondence to: Dr Nadel; s.nadel@ic.ac.uk

R Booy

Department of Child Health, Queen Mary's School of Medicine and Dentistry, University of London, UK

\section{References}

1 Petros AJ, Weir P, Marsh M, et al. Genuine reduction in meningococcal deaths results from teamwork. [electronic response to Booy et al, Reduction in case fatality rate from meningococcal disease associated with improved healthcare delivery]. www.archdischild.com http:// adc.bmijournals.com/cgi/eletters/ archdischild;85/5/386 laccessed 7 March 2002) 
2 R Booy, P Habibi, S Nadel, et al. Reduction in case fatality rate from meningococcal disease associated with improved healthcare delivery. Arch Dis Child 2001;85:386-90.

3 Tilford JM, Robertson PK, Lensing S, et al. Differences in paediatric ICU mortality risk over time. Crit Care Med 1998;26:1737-43.

4 Derkx B, Wittes J, McCloskey R, et al. Randomized placebo-controlled Trial of HA-1A, a human monoclonal antibody to endotoxin, in children with meningococcal septic shock. Clin Infect Dis 1999;28:770-7.

5 Levin M, Quint PA, Goldstein B, et al. Recombinant bactericidal/

permeability-increasing protein $(\mathrm{rBPI} 21)$ as adjunctive treatment for children with severe meningococcal sepsis: a randomised trial rBPI2 1 Meningococcal Sepsis Study Group. Lancet 2000;356:961-7.

\section{Why do infants being treated for acute lymphoblastic leukaemia fail to thrive?}

Figure 1 shows the weight gain of five infants treated for acute lymphoblastic leukaemia (ALL) in relation to centile chart positions, who were treated at the Yorkshire Regional Centre for Paediatric Oncology and Haematology from 1996 until the present. Patients 1-4 were treated in accordance with the MRC UKALL Infant 1 chemotherapy protocol, ${ }^{1}$ and patient 5 in accordance with Interfant $99 .^{2}$

The most striking aspect is that from diagnosis to end of intensive therapy (approximately week 40 of treatment), the first four patients, despite aggressive nutritional support, failed to thrive, with two requiring long term total parenteral nutrition (TPN) during Patient 5, in contrast, thrived during treat ment.

The infants treated on the MRC UKALL Infant 1 protocol all had grade III/IV gut toxicity following intensive therapy, resulting in the infants being highly catabolic; although some weight gain was achieved with TPN, it was difficult to sustain this increase with enteral feeding.

It became apparent that patients not fully weaned at diagnosis showed a severe delay in feeding skills, becoming orally defensive, resulting in a grossly inadequate solid intake throughout intensive treatment, which continued into maintenance therapy. The only maintenance therapy in order to rectify this.

patient to continue normal feeding development was patient 4 , who was 36 weeks at diagnosis, and fully weaned.

Patient 5, like the others diagnosed under 30 weeks old, had delayed feeding skills, taking virtually no solids or feed orally. However she did not show such severe gut toxicity. She was fed an amino acid based formula (Neocate, SHS International Ltd, UK) since induction therapy.

The failure of infants with ALL to thrive may be consequent on severe gut toxicity length of treatment, and failure of weaning. The improved outcome of patient 5 may be the result of use of a different chemotherapy protocol, which included dexamethasome.

A second possibility is the early introduc tion of an amino acid based formula, which is a source of L-glutamine, an important nitrogen source for enterocytes, which plays a key role in maintaining mucosal cell integrity and gut barrier function. ${ }^{3}$ It may be that exposure to a continuous low dose of glutamine throughout intensive chemotherapy helped to reduce the severity of mucositis.

E Ward

Dietetic Department, St James's University Hospital Beckett Street, Leeds LS9 7TF, UK paediatric_dietitian.sjuh@leedsth.nhs.uk

S Kinsey, M Richards

Yorkshire Regional Centre for Paediatric Oncology \& Haematology

\section{References}

1 Medical Research Council. UKALL Infant 1 protocol, 1992.

2 Medical Research Council/United Kingdom Children's Cancer Study Group. Interfant 99, 1999

3 Hall JC, Heel K, McCauley R. Glutamine. Br J Surg 1996;83:305-12.

4 Miller AL. Therapeutic considerations of L-glutamine: a review of literature. Altern Med Rev 1999:4:239-48.

\section{Osteogenesis imperfecta and intravenous pamidronate}

Osteogenesis imperfecta (OI) is a chronic, disabling condition in which treatment with cyclical intravenous treatment with pamidronate can be useful for symptom relief, ${ }^{1}$ despite questions about long term safety. ${ }^{2}$ A recent

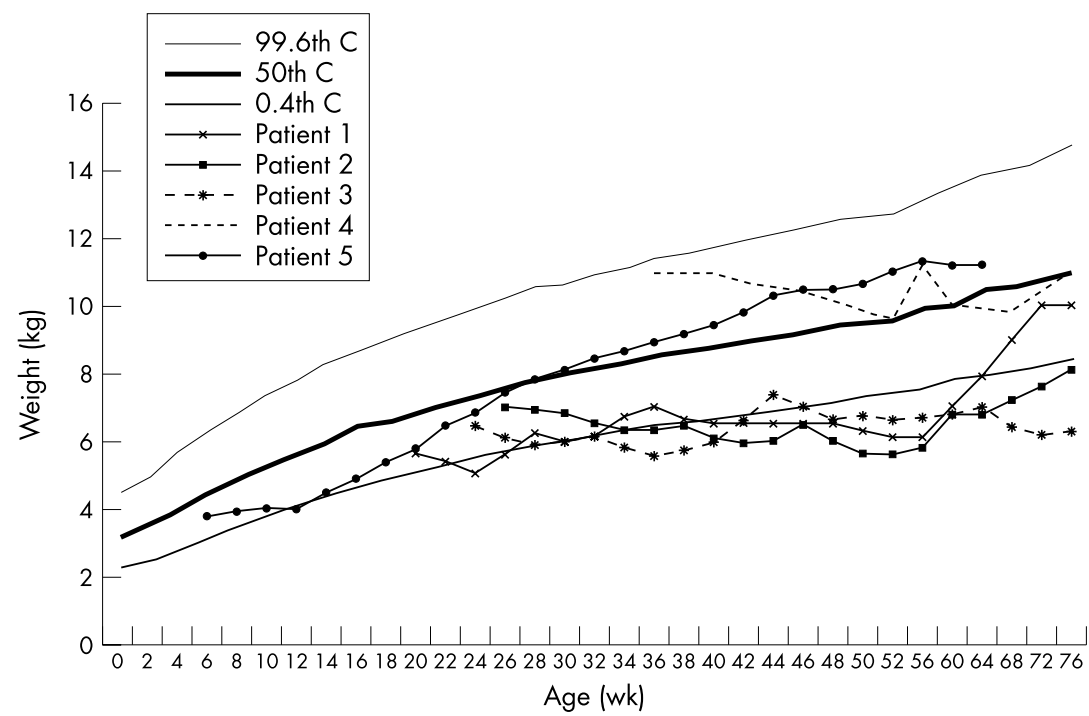

Figure 1 Weight gain since diagnosis in five infants with ALL, showing peaks and troughs related to intensive treatment blocks.

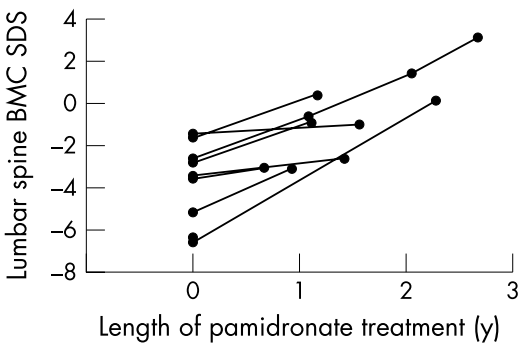

Figure 1 Effect of pamidronate on lumbar spine BMC.

study $^{3}$ in this journal showed a decrease in bone turnover and gradual increase in bone density measurements without significant side effects, following such treatment in children affected with OI.

We wish to report our own experience in 10 children with OI who have received cyclical intravenous pamidronate $(1 \mathrm{mg} / \mathrm{kg} /$ day for three days every three months). The median (range) age at the start of treatment was 9.1 (1.3-12.7) years. Treatment was initiated in the context of symptoms having an adverse effect on the quality of life, associated with evidence of decreased bone density assessed by dual energy $x$ ray absorptiometry (DXA scan, QDR1000/W, Hologic systems, Boston, Massachusetts). ${ }^{4}$

Five of these children were at the severe end of the symptom spectrum (recurring pain, multiple fractures, and impaired mobility). Four children were treated for pain and fractures, whereas one received pamidronate for pain only. After 1.8 (0.9 to 3.0) years of treatment, nine children were pain free. Four children had had no further fractures and one child had improved mobility. The initial infusion of pamidronate was associated with flu like symptoms, fever, rigors, abdominal pain, or vomiting in six children. Serum calcium levels were low $(<2.2 \mathrm{mmol} / \mathrm{l})$ following therapy in six subjects, and three required treatment with calcium and vitamin D supplements.

Repeat DXA scans showed an increase in lumbar spine bone mineral content (BMC) standard deviation score (SDS) (fig 1), from $-3.44(-6.6$ to -1.39$)$ to $-0.96(-3.10$ to 3.13 ) SDS following 1.3 (0.7 to 2.7 ) years of pamidronate treatment. This beneficial response to treatment was similar to that reported elsewhere. ${ }^{3}$

In conclusion, our findings add to those of others that intravenous pamidronate infusion improves bone mineral density and reduces symptoms of severe pain, recurrent fractures, and impaired mobility in children with OI. There are minor acute side effects to the treatment, but long term safety needs to be determined.

I Baneriee, G J Shortland, W D Evans Department of Child Health, University Hospital of Wales, Heath Park, Cardiff CF 14 4XW, UK; ibanerjee@freeuk.com

J W Gregory University of Wales, College of Medicine, Heath Park, Cardiff CF1 4 4XN, UK

\section{References}

1 Glorieux FH, Bishop NJ, Plotkin $\mathrm{H}$, et al. Cyclic administration of pamidronate in children with severe osteogenesis imperfecta. N Engl J Med 1998;339:986-7.

2 Shaw NJ, Bovin CM, Crabtree NJ. Intravenous pamidronate in juvenile osteoporosis. Arch Dis Child 2000;83: 143-5. 
3 Alstrom E, Soderhall S. Beneficial effect of long term intravenous bisphosphonate treatment of osteogenesis imperfecta. Arch Dis Child 2002; 86:356-64

4 Warner JT, Cowan FJ, Dunstan FD, et al. Measured and predicted bone mineral conten in healthy boys and girls aged $6-18$ years: adjustment for body size and puberty. Acta Paediatr 1998;87:244-9.

\section{Growth charts for height and weight-statement}

The recent review by Professor Noel Cameron $^{1}$ was, at least in our view, the most balanced of a number of reports published in recent times.

However, on reading the introduction and historical background to this article, we were once again surprised to find that the Tanne Whitehouse Takaishi (1966) and the Tanner Whitehouse (1976) height and weight chart (copyright Castlemead Publications), are both discussed as being widely used in hospitals, departments of paediatrics, community health departments, and academic institutions.

We say "once again surprised", as Professor Cameron's review, despite being so well balanced, is the latest in a series of articles, reviews, and publications stretching back ove a number of years which uses either one or other, or both, of the above stated charts as references when discussing or comparing growth charts. ${ }^{2-5}$ Indeed, one or two of these previously published articles have been wholly misleading, comparing as they did the British 1990 reference with the above, while at the same time failing to mention the introduction of the updated Buckler-Tanner (1995) (Castlemead Reference 11B and 12B).

The fact that the Tanner Whitehouse Takaishi (1966) and Tanner Whitehouse (1976) charts still appear to be readily available is indeed a source for concern, as none of the former have been produced or sold by ourselves for an absolute minimum of 15 years (our sales records go no further back) while the latter has neither been produced or sold for a period of some seven years. In short, Castlemead fully accept that the Tanne Whitehouse Takaishi (1966) and Tanner Whitehouse (1976) are long since obsolete, and should play no part in any considerations respecting growth charts for height and weight. Given the above, we are at a loss to understand why these two charts continue to appear as subjects for review.

In an attempt to draw this particular issue to a close, Castlemead is prepared to offer any hospital, department of paediatrics, community health department, or academic institution still holding stocks of either the Tanner
Whitehouse Takaishi (1966) or Tanner Whitehouse (1976) (Castlemead Reference 11A and 12A) a "new" for "old" replacement of their stock with the updated Buckler Tanner (1995) growth charts (Castlemead Reference 11B and $12 \mathrm{~B}$ ).

P Wraith

Castlemead Publications, Hertford, UK peter@castlemeadpublications.com

\section{References}

1 Cameron N. British growth charts for height and weight with recommendations concerning their use in auxological assessment. Ann Hum Biol 2002; 29: 1-10

2 NHS Executive Child Health in the Community. A guide to good practice. 6.27. NHS Executive September 1996:35.

3 The Child Growth Foundation. Growth assessment charts. Guidelines in practice, Volume 10. February 2000:193.

4 Rudolf MC, Cole TJ, Krom AV, et al. Growth of primary school children: a validation of the 1990 references and their use in growth monitoring. Arch Dis Child 2000;83:298-301.

5 Wright CM, Booth IW, Buckler JMH, et al. Growth reference charts for use in the United Kingdom. Arch Dis Child 2002;86:1 1-14.

\section{Juvenile dermatomyositis associated with hereditary angioneurotic oedema}

Juvenile Dermatomyositis (JDM) is a chronic inflammatory disease probably of an autoimmune nature. ${ }^{12}$ Hereditary angioneurotic oedema (HANE) is an enzyme deficiency that results in the loss of inhibition of the classical complement pathway. This results in the consumption of classical pathway factors particularly C4. It is associated with some autoimmune disorders, particularly SLE. We report for the first time the occurrence of JDM in a child with HANE.

A 6 year old Caucasian boy with a family history of type 2 HANE presented with a 4 month history of a red, scaly rash on the back of his fingers and hands, on the dorsum of his feet and toes, on his knees, and above both eyelids. The rash appeared characteristic of JDM. He had difficulty in climbing stairs. Clinical examination revealed some weakness of the proximal muscles. Investigations included a raised creatine kinase of $3000 \mathrm{U} / \mathrm{l}$. (normal 50-150), a muscle biopsy typical of JDM, very low levels of C4 and CH50, and confirmation of type 2 HANE with absen functional $\mathrm{Cl}$ inhibitor activity but raised immunochemical levels. Complement C4 returned to normal levels after 2 months treatment with danazol but there was no change in the clinical or laboratory signs of dermatomyositis. Complete resolution of the clinical and biochemical signs of myositis occurred a short time after the introduction of prednisolone The danazol was stopped but the prednisolone was continued. The reduction in serum complement C4 returned but there has been no clinical deterioration. Subsequently the prednisolone was stopped and there has been no flare of his JDM.

Interestingly the administration of danazo to patients with SLE and HANE has led to the reduction in complement consumption and thus normalisation of $\mathrm{C} 4$ levels in the classical pathway accompanied by resolution of the SLE. ${ }^{3}$ However no such effect was seen in our patient. The failure to alter the course of our patient's JDM by restoration of the classical pathway components is interesting. It does not suggest that the aetiology of JDM is due to failure of clearance of immune complexes. However, it is possible that the uncontrolled classical pathway activation or acquired C4 deficiency may have contributed to the initiation of the disease.

R Narasimhan, R Lakshman, R S Amos, L H P Williams

Bassetlaw District General Hospital, Worksop, UK

W Egner

Northern General Hospital, Sheffield, UK

A Finn

University of Bristol Medical School, Bristol, UK

Correspondence to: Dr Williams;

Catherineandleonard.Williams@btopenworld.com

\section{References}

1 Albani S. Infection and molecular immunity in autoimmune diseases of childhood. Clin Exp Rheumatol 1994; Suppl 10:S35-41.

2 Plotz PH, Rider LG, Targoff IN, et al. NIH Conference. Myositis: Immunologic contributions to understanding cause, pathogenesis, and therapy. Ann Intern Med 1995; 122:715-24

3 Donaldson VH, Hess EV. Effect of danazol on lupus-erythematosus-like disease in hereditary angioneurotic oedema. Lancet 1980;2: 1145

4 Masse R, Youinou P, Dorval JC, et al. Reversal of lupus-erythematosus like disease with danazol. Lancet 1980;2:651.

\section{CORRECTION}

In the paper by Kneen et al in the September issue of Archives (Arch Dis Child 2002;87:181$3)$, figure 1 was reproduced with permission from reference 8 (Solomon $\mathrm{T}$, Kneen $\mathrm{R}$. Neurological Presentations. In: Beeching N, Gill G, eds. Lecture Notes on Tropical Medicine. Oxford: Blackwell Science, 2002 (in press), and not reference 6 . The journal apologises for the error. 